\title{
Erratum to: Liquid-injection for preperitoneal dissection of transabdominal preperitoneal (TAPP) inguinal hernia repair
}

\author{
Tomoko Mizota $\cdot$ Yusuke Watanabe $\cdot$ Amin Madani \\ Norihiro Takemoto $\cdot$ Hidehisa Yamada $\cdot$ Saseem Poudel $\cdot$ \\ Yuji Miyasaka $\cdot$ Yo Kurashima
}

Published online: 10 January 2015

(C) Springer Science+Business Media New York 2015

\section{Erratum to: Surg Endosc}

DOI 10.1007/s00464-014-3703-7

In the title the word "inguial" is correctly spelled "inguinal".

On the first page, in the Abstract, 8th line, "preperitonal" should be "preperitoneal".

At the bottom of the left column, first page,

T. Mizota, N. Takemoto, H. Yamada, Y. Miyasaka

The online version of the original article can be found under doi:10. 1007/s00464-014-3703-7.

T. Mizota $(\bowtie) \cdot$ N. Takemoto $\cdot$ H. Yamada $\cdot$ Y. Miyasaka NTT East Sapporo Hospital, Sapporo, Hokkaido, Japan e-mail: tmizota@huhp.hokudai.ac.jp

N. Takemoto

e-mail: nori-take@pop16.odn.ne.jp

H. Yamada

e-mail: h-yamada@east.ntt.co.jp

Y. Miyasaka

e-mail: cs-miya@east.ntt.co.jp

T. Mizota $\cdot$ Y. Watanabe $\cdot$ S. Poudel $\cdot$ Y. Kurashima

Department of Gastroenterological Surgery II, Hokkaido

University Graduate School of Medicine, North 15 West 7,

Kita-ku, Sapporo 060-8638, Hokkaido, Japan

e-mail: ywatanabe328@gmail.com

S. Poudel

e-mail: saseem@gmail.com

Y. Kurashima

e-mail: yo.kurashima@huhp.hokudai.ac.jp

\section{A. Madani}

Department of Surgery, McGill University, Montreal, QC, Canada

e-mail: amin.madani@mail.mcgill.ca
NTT East Sapporo Hospital, Sapporo, Hokkaido, Japan e-mail: nori-take@pop16.odn.ne.jp

should be changed to

T. Mizota $\cdot$ N. Takemoto $\cdot$ H. Yamada $\cdot$ Y. Miyasaka NTT East Sapporo Hospital, Sapporo, Hokkaido, Japan e-mail: tmizota@huhp.hokudai.ac.jp

N. Takemoto

e-mail: nori-take@pop16.odn.ne.jp

On the second page, left column, 4th paragraph, first line, delete "to".

On the fifth page, left column, first paragraph, $9^{\text {th }}$ line, change "safely" to "safety".

In Reference 8, change "Naoki M" to "Matsumura N". 